\title{
Historic cities and sustainable tourism: a configurational approach
}

\author{
V. Cutini \\ University of Pisa, Italy
}

\begin{abstract}
Tourism, as aimed at visiting and experiencing unknown places, obviously involves several questions regarding the use and management of urban space: where to locate bus terminals and car parking, how to guide and orient visitors in the grid of urban paths towards monuments and amenities, how to enhance their spatial comprehension, how (and where) to assist them with services and facilities, how (and where) to improve accessibility for all sorts of people, to mention the most evident. Such issues will be faced herein using a configurational approach, thus assuming the urban grid as the primary element in the phenomena along its paths, and in particular the distribution of movement and the location of activities. The configurational approach, whose reliability has been widely attested by a large number of studies in the last two decades, appears particularly suitable for supporting the touristic realization of cities, as a consequence of the fact that tourists are assumed to be non-expert urban users, generally unaware of the inner geography and of the functional features of a settlement and thus strongly moderating their behaviour from the perception and understanding of the surrounding spatial features. Furthermore, it ought to be noted that most of the historic centres of cities, renowned destinations of tourists from all over the world, are actually characterized by highly irregular and often labyrinth-like urban grids, sometimes confusing and barely accessible, so as to demand some comprehension and support during visits. The purpose of this research is to pinpoint the way a configurational approach can actually be used to improve touristic accessibility and to enhance the ease and safety in the realization of tourists visiting historic cities.
\end{abstract}

Keywords: tourism, orientation, accessibility, grid configuration. 


\section{Introduction}

The touristic visit of cities can actually be regarded as a peculiar urban experience, in that the presence of non-expert urban users aimed at exploring an unknown urban environment recalls and amplifies several questions concerning urban knowledge and management. First (and most obvious), how and where to locate tourist services and facilities (bus terminal and car parking, toilets, information points, etc.) in order to simplify visits and assist visitors. Then (and less obvious) how to facilitate and enhance an accurate comprehension of urban space, so as to make tourists well aware of the spatial consistency of the settlement and able to consciously choose their paths towards their chosen destinations, thus easily orienting within the urban grid. Moreover (not so obvious) how to improve urban accessibility for all sorts of people (including children, the elderly or single persons, etc.) so as to eliminate any discrimination and extend the potential range of visitors. Furthermore (and not obvious in the least) how the location and management of services and facilities can help to provide a key for the access and visit of cities, possibly modifying their intrinsic disposition. All these questions are generally faced by means of an interactional approach: given the position of tourist terminals (bus parking, railway station) and tourist attractions, the question is to allow/rationalize/optimize their mutual interaction. So far, several studies, recent and refined [1], have been carried out on these issues. Yet, this kind of approach has serious limits, in that it assumes that urban space is a mere object and can hence hardly account for the role that space itself plays in the distribution of tourist movement flows and in the location of any kind of activities. In other words, tourists are generally unaware of the inner geography and functional consistency of a settlement (distribution of activities and land use) and therefore strongly define their behaviour from what they actually perceive and understand of the surrounding spatial features; what an interactional approach can hardly appreciate, account for and manage. Moreover, in several cases tourist attractions can hardly relate to pinpoint destinations, rather appearing scattered all over a whole and wide historic context [2]. Furthermore, most of the historic cities, renowned touristic destinations, are characterized by irregular and often labyrinth-like urban grids, somehow confusing and hardly accessible, so as to strongly demand some understanding and support for the visit [3].

The perception of urban space does hence play a role, which can hardly be comprehended and managed by means of traditional interactional methods. This incapability is pretty clear in the actual accessibility and visiting potential of many Italian historic cities (Venice above others), which can hence be assumed to be persuasive examples. Other methods have been introduced and tested [4] in order to overcome such limits and to go beyond a mere deterministic approach, using multi-agent systems and agent-based modelling.

In the present research urban space comes into play. The above mentioned issues will be faced using a configurational approach, so as to assume the urban grid as the primary element in the phenomena along its paths, and in particular the distribution of movement and the location of activities [5]. It will 
be shown - and tested on several case studies - how a configurational approach can guide the location of services and facilities to improve urban accessibility and to enhance the ease and safety in the realisation of historic cities.

As a testing ground of such an approach, several Italian urban centres (Florence, Lucca, Pisa, Positano, San Gimignano, Siena, Venezia, Volterra), quite different from each other on any regard, were assumed as case studies, in order to highlight specific problems, to propose support tools and, even more, to apply and test a general operational method.

\section{Tourism and configurational approach}

The configurational approach to the analysis of urban settlements, introduced in the mid 1980s by Hillier and Hanson [6] and since then applied, improved and developed by large teams of researchers all over the world, is based on the role of the urban grid as the primary element in the phenomena occurring along its paths, in particular movement distribution and location of activities. The idea is that a portion of movement, called 'natural movement', does not depend on the presence and position of activities but only on the spatial relations between its elements, that is, the grid configuration [7]. It's the grid configuration that indicates and suggests the likely distribution of movement flows and hence the pre-condition for the use of urban land. On such basis, several operational techniques have so far been introduced and developed. The first, introduced by Hillier and Hanson in 1984 [6], is the axial analysis, which transforms the grid into a system, called an axial map, composed of the fewest and longest lines that cover all the grid, connecting all its convex spaces. Other techniques, including visibility graph analysis [8], angular analysis [9], segment analysis [10], mark point parameter analysis [11], sharing the same conceptual basis, differ from one another by the way of reducing the grid into a system, and actually present advantages and limits, so as to coexist, suitable for being used in different cases and with different purposes [12].

All those operational methods provide each element of the system with a full set of configurational variables. Among them, connectivity (the number of elements directly connected to the observed one) and integration (the mean depth of the observed element with regard to all the others) deserve particular consideration. The relevant significance of integration derives from several studies, which in the last decades have proved that it is suitable for narrowly reproducing the actual distribution of urban centrality, meant as the measure of attractiveness towards movement and activities [13]. Such notion of centrality can be said to be pure, in that it does not depend on the located activities, but is merely determined by the spatial relationships between the elements, that is by the grid configuration. In other words, the distribution of integration narrowly reproduces the distribution of the positional appeal of the spatial elements of the grid. Some peculiar features actually make the configurational approach specifically suitable for facing and overcoming the above mentioned questions regarding the touristic use of cities. 
First, the assumption of the grid as the primary element of many urban phenomena actually provides the space of a settlement with a foreground role, and allows configurational analysis to face questions concerning the morphology of urban texture. In fact, configurational techniques assume the urban grid with its material consistency (from the pattern of the streets up to the shape of blocks and buildings) as their input variable, so as to provide results that regard several aspects of the working of the settlement. More concrete, such a feature makes them suitable for predicting the effects of any material transformation of the grid on a wide range of material and unimportant urban variables (distribution of flows, distribution of land values, accessibility, etc.).

The thesis is that urban space, by means of the visual perception of visitors, actually plays a role. It orients, guides and addresses the movement flows, enhances the positional value of single places while segregating and hiding others. Only a deep understanding of this role can hence support conscious and sustainable tourism. Tools for investigating this role are therefore definitely recommended: what configurational analysis precisely provides.

In further detail, the idea is that the distribution of some configurational indices can strongly support the touristic use of ancient urban centres in several respects: in order to locate information points and facilities, to select the paths to be made accessible [14], to match the visit of places with the presence and operation of local activities, so as to favour actual tourism sustainability.

\section{Methodology}

In this paper, three applications of configurational techniques, narrowly correlated and integrated one with another, are briefly outlined and proposed as suitable tools for supporting, improving and managing the touristic accessibility of historic centres.

A first application concerns the understanding of the degree to which an urban grid can be said to be labyrinthian, which actually confuses and disorients any visitor, making him need guidance and support. This aspect will hence allow us to understand (and comply with) the need for tourism assistance and support that an urban grid actually requires. Another application will then regard the location choice of services and facilities, in order to make accessibility cope with the actual distribution of movement and visitors. This aspect will therefore be aimed at rationalizing and optimizing resources for visits and tourism. The last one concerns the possibility of managing the distribution of touristic flows, influencing it by means of the location of touristic services, thus defining the distribution determined by the grid configuration.

Each of those three applications clearly focuses on urban space, recognizing it as the primary element in urban phenomena, including touristic visits to urban places and attractions. It's then the physical space of the settlement, assumed to be the main input variable of the model that is expected to provide information on its touristic accessibility. More generally, the city is examined on its availability to be visited, insofar as it results from the position, shape and relationships of its paths; as shown in the following. 
A first, and somehow rough, indicator of the level of confusion and disorientation that a grid is actually likely to provide is the mean value of connectivity of its elements. Since connectivity varies from 1 (elements with a unique connection, that is cul-de-sac elements) and n-1 (element connected to all the other elements of the grid, being $\mathrm{n}$ its total consistency), a low value of connectivity is expected to stand for a strong presence of obliged paths, thus poorly depending on space perception. Conversely, high values of connectivity suggest a wide range of path choices, according to the perception of intermediate destinations. In this regard, the labyrinth (Figure 1) and the panopticon can be seen as opposite paradigms. Each step along a labyrinth is governed by the preceding one and governs the next step, no matter the destination, which cannot be seen. Conversely, each path on a panopticon is composed of a one-step path, from a source to the destination, which is in full view. The mean value of connectivity in a labyrinth is $\mathrm{C}_{M}=2(\mathrm{n}-1) / \mathrm{n}$, varying from 1 to 2 and approaching 2 as $n$ grows, while in a panopticon $C_{M}=n-1$, thus approaching $n$. A panopticon is not really transferable into an urban grid, but can be somehow approximated by the fortress-city of Palmanova (Figure 1), provided with $C_{M}=20.9$.

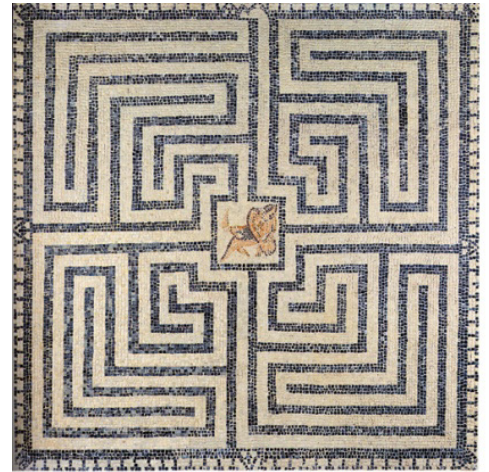

(a)

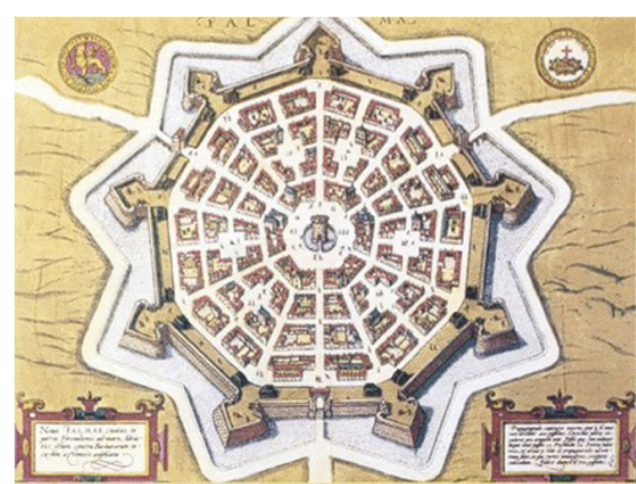

(b)

Figure 1: The labyrinth (a) and Palmanova (b), opposite archetypes of intelligibility.

A further indicator of the same feature is the determination coefficient $\mathrm{R}^{2}$ of the correlation of integration versus connectivity, in the literature generally called intelligibility. A high value of intelligibility means that the most connected spaces are also the most integrated, and hence that the more accessible spaces give access to a larger number of other spaces. Recalling the above mentioned references, a labyrinth is provided with a very low (almost zero) intelligibility value, while in Palmanova $\mathrm{R}^{2}=0.98$.

A third indicator is then the correspondence of local integration with global integration, realised by the $\mathrm{R}^{2}$ coefficient of radius 3 integration (computed taking into account the lines within a local area) versus radius $\mathrm{n}$ (extended all over the urban area). The idea is that the local spatial perception actually corresponds to the global geography of the settlement, and is hence suitable for 
guiding the paths all over the grid. Once more, the findings confirm such assumption, with $\mathrm{R}^{2}=0.02-0.04$ in a labyrinth and $\mathrm{R}^{2}=1.00$ in Palmanova.

Summing up, the three parameters indicated above can be used in order to evaluate the level of intelligibility with which an urban settlement is intrinsically provided, as a consequence of its spatial features, and hence the actual need of support and assistance to users and visitors. This application could be named 'configuration analysis for grid comprehension'.

The second application proposed herein is aimed at supporting the location of services and facilities, so that they correspond to the actual distribution of tourist movement flows. Generally, this question is faced by taking into account the specific position of tourist attractions as well as the location of transport terminals (railway stations, bus and car parking). Yet two aspects ought to be considered. First, tourism attractions rarely are concentrated and punctiform, so as to be regarded as pinpoint destinations. More often they are uniformly scattered all over the grid of the historic centre, so that it's actually difficult (when not actually impossible) to regard them as single pivot points in pedestrian touristic movement. Even in the particular case of Pisa, characterized by the presence of the singular worldwide attraction of the Leaning Tower, its assumption as a single terminal of movement paths could cause a failure to capture the actual visitor flows, as well as disregarding the whole historic context that surrounds it and the tourist attractions within the ancient town-walls. Something that unfortunately does occur in Pisa in the present distribution of touristic services. Within a historic city, therefore, the location of touristic services would better be located with reference to the distribution of natural movements (depending on the configuration of the grid) rather than on the mere basis of the position of a single attraction. It's then the distribution of the configurational indices of centrality that appears likely to reproduce the distribution of movement, and hence the indication for the location of services and facilities. Moreover, the same distribution of centrality can also provide a valuable hint concerning the location of touristic transport terminals and services, citing them (for instance, bus and car terminals, or public transport lines) near the most crowded areas of the settlement. This application could be named 'configuration analysis for grid accessibility' and also provides indications on the relationships between the distribution of natural movements and touristic flows, useful for managing the coexistence of tourism and ordinary economic activities.

The third application is subtler and apparently nearly opposite to the preceding one. It uses this approach not to more efficiently locate touristic services with reference to the touristic flows, but to locate touristic services (in particular transport terminals) in order to guide and manage the distribution of touristic flows. It is subtler, in that it is not aimed at enhancing the accessibility of areas visited by tourists, but at managing their magnitude, defining the movement distribution resulting from the configurational analysis of the grid. More generally, the use of configurational techniques highlights the distribution of natural movements, determined by the grid configuration, which will not overlap touristic movements, depending on the position of transport terminals, as 
sources, and touristic attractions, as destinations. The location of sources appears to be a tool for managing the relationships between natural movement and touristic flows (either making them coincide or separating them), therefore the relationship between the touristic realization of cities and the working of local activities. In other words, the grid configuration involves the distribution of touristic flows, which can be modified (for any reason, for instance, to guide tourists towards hidden or neglected areas) by the location of tourism services. For a better comprehension of this issue, a historic city can be usefully conceptualized as an open air museum. The internal organization of museums and exhibitions spaces has been widely studied and discussed, in order to increase their accessibility and to improve the realization of the collected pieces, and space syntax techniques have been tested and proved suitable for supporting it [15-17]. The obvious difference is that our open air museums generally don't allow the displacement of attractions, so that the only variables in their arrangement are the spatial grid and the location of services. That is precisely the purpose of this last application, which could be named 'configuration analysis for grid management'.

\section{Case studies}

The three operations so far presented were applied to several historic cities, corresponding to well-known Italian touristic destinations, taken as case studies. Here the outcome of such experimentations, with reference to the cases of Pisa, Lucca and Venice, will be presented and briefly discussed. What makes them suitable for a specific discussion is the fact that they are approximately a similar size (both physical and demographic) and conversely have radically different spatial features, with regard to the issue of accessibility and orientation. Such differences arise from the results of the grid configuration analysis, which will be here presented with reference to the three applications outlined above.

First, concerning the configurational analysis for grid comprehension, a comparison of the three parameters taken to reproduce the level of intelligibility is presented in Table 1, and appears worth discussing.

Table 1: Values of the three proposed parameters in Lucca, Pisa and Venice.

\begin{tabular}{|c|c|c|c|}
\hline & Lucca & Pisa & Venice \\
\hline $\mathrm{C}_{\mathrm{M}}$ & 5.53 & 7.43 & 2.71 \\
\hline $\mathrm{I}_{\mathrm{n}} / \mathrm{C} \mathrm{R}^{2}$ & 0.38 & 0.46 & 0.01 \\
\hline $\mathrm{I}_{3} / \mathrm{I}_{\mathrm{n}} \mathrm{R}^{2}$ & 0.64 & 0.63 & 0.06 \\
\hline
\end{tabular}

The results clearly show that the two cases of Pisa and Venice can be taken to be paradigmatic. On the basis of the resulting values, Pisa appears highly understandable and Venice, on the contrary, markedly not understandable so as to confuse tourists and unfamiliar visitors. With regard to the other cities, Lucca 
appears in an intermediate position, in that all the parameter results are included between them. For a detailed view, the exponential correlation of connectivity versus integration is here represented in Figure 2, so as to highlight the differences above.
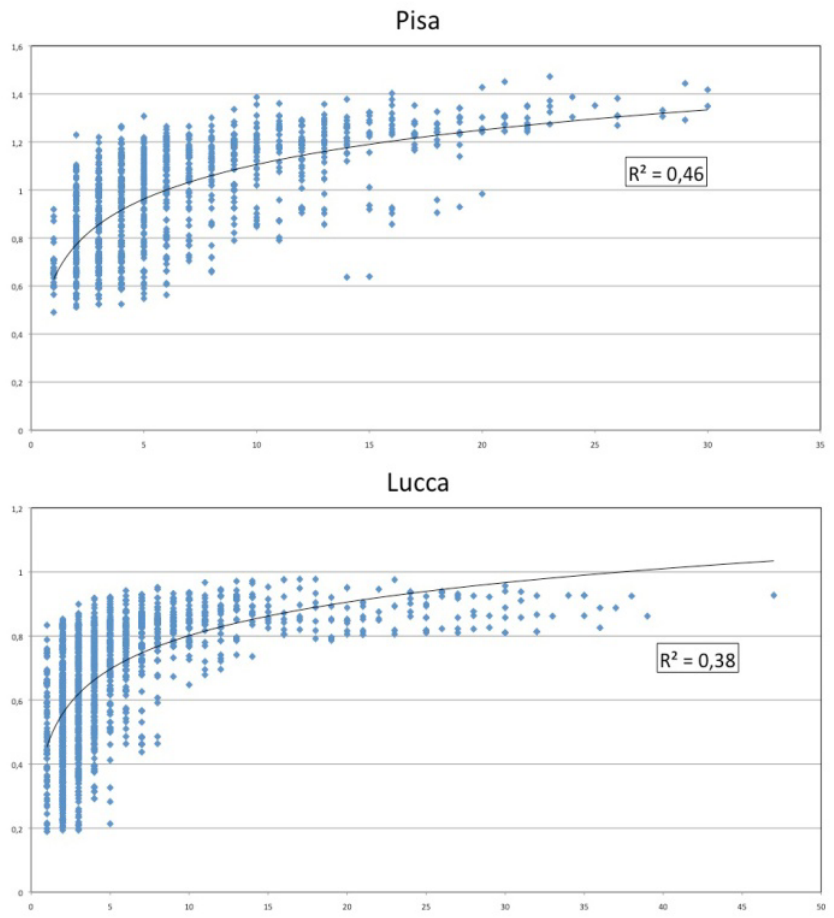

Venezia

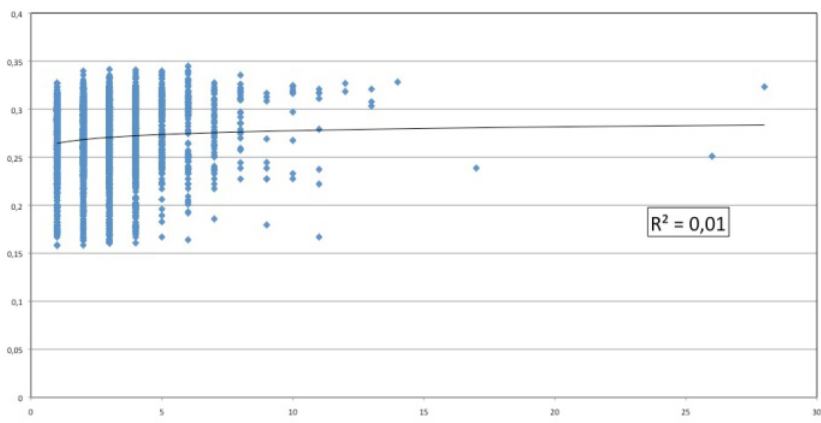

Figure 2: Connectivity versus global integration (intelligibility) in Pisa, Lucca and Venice.

Also the configuration analysis for grid accessibility provides interesting results when applied to the cities above. In the case of Pisa, shown in Figure 3, the lines providing the highest integration values (over the 95th percentile, 
comprising the so-called 'integration core') shape a rough cross centred on the main bridge (Ponte di Mezzo) and oriented north-south. This was proved [18] to narrowly correspond to the actual distribution of pedestrian movement.

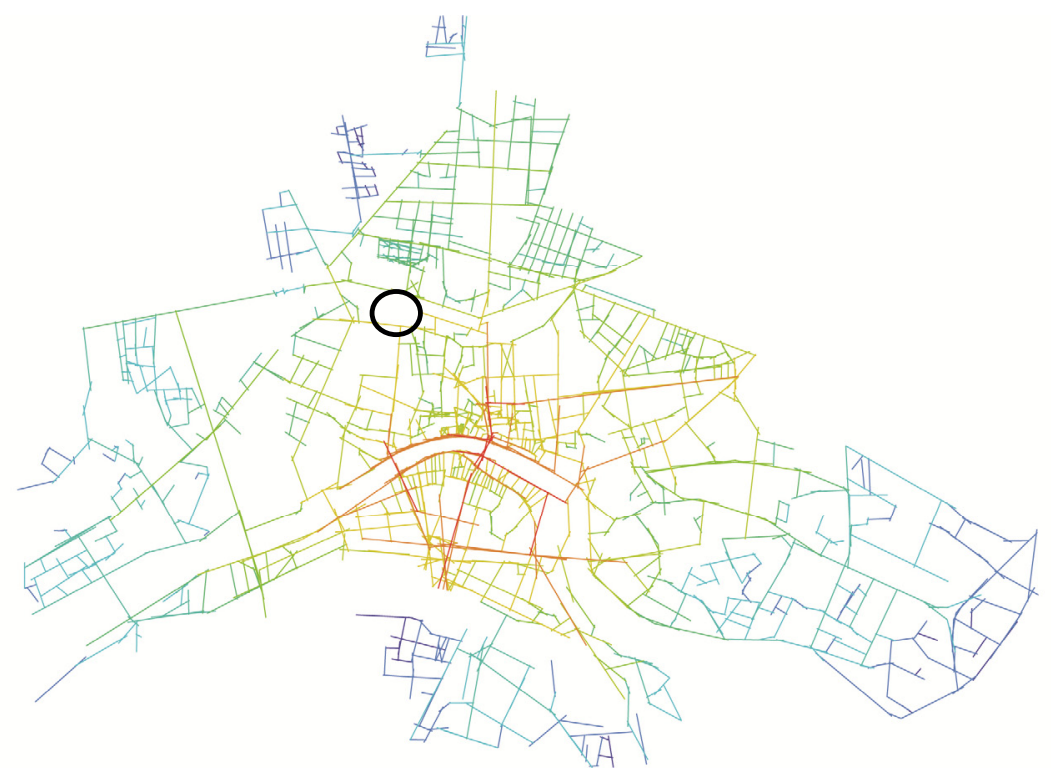

Figure 3: The distribution of global integration value in Pisa. The position of the Leaning Tower is circled.

The main attraction of Piazza dei Miracoli appears in contrast to be in a poorly integrated position. This result highlights two problems with tourism in Pisa. On one hand, tourists generally struggle to find the Leaning Tower, their main destination, as it is beyond the most crowded paths. On the other hand, most tourists that annually visit the Tower, reach it by coach or private car, and remain outside the historic centre of Pisa, thus missing its activities and disregarding a number of prominent attractions. In this regard the case of Venice (Figure 4) is quite different. Here the crowded touristic paths (mainly connecting Santa Lucia railway station, the Rialto and Piazza San Marco) appear to follow the routes to the centre, and visitors' movements hence coincide with the natural ones. These results show that, in Pisa, development in touristic information is strongly advised in order to address pedestrian flows towards the main attractions. In Venice, on the contrary, services are mainly demanded along the mentioned touristic axis, and services are advised in order to suggest and propose alternative targets as well as to orient visitors away from the main touristic flows [18].

Despite the differences so far discussed, in all the cases the use of configuration analysis for grid management is advised, for different reasons. In Venice the location of services (in particular transport services, such as vaporetto 
or traghetto) can usefully define the distribution of touristic flows so as to involve other destinations apart from the traditional targets of Piazza San Marco and the Rialto.

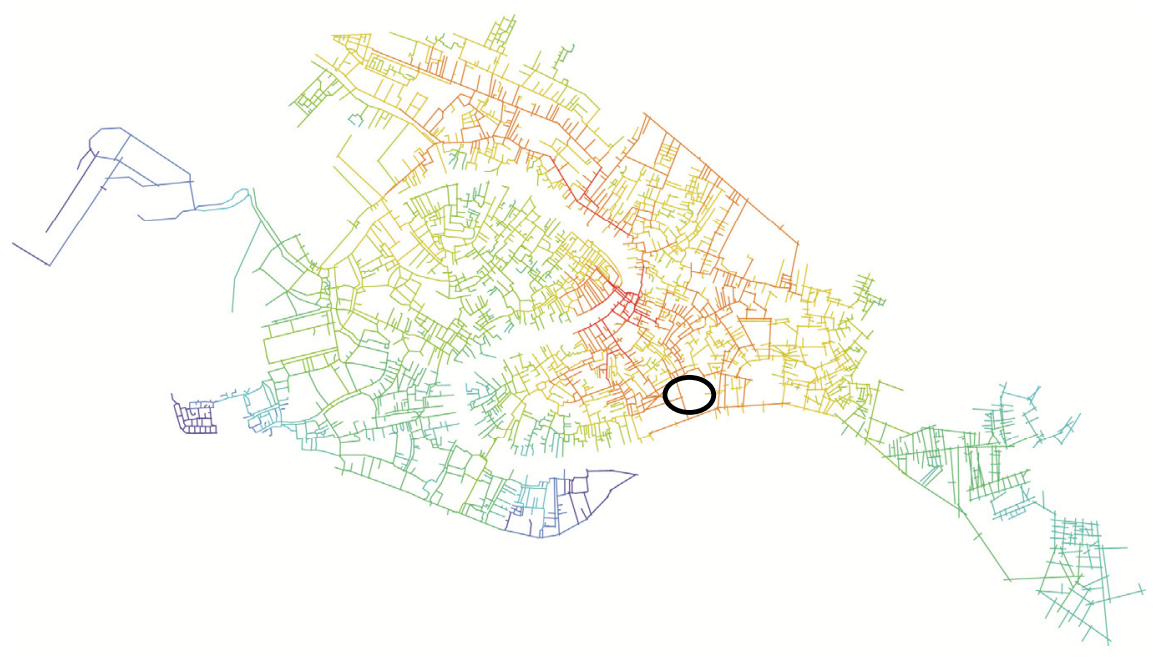

Figure 4: The distribution of global integration value in Venice. The position of Piazza San Marco is circled.

In Pisa the present location of coach terminals and car parking, on the western edge of the city, actually confines most tourists to merely visiting the Tower, thus ignoring the remaining areas of the inner centre. Shifting that location could drive touristic flows to coincide with the distribution of natural movement, thus involving the whole historic city. In Lucca, which actually lacks a prominent touristic focal point, attractions are scattered all over the historic fabric. From a configurational point of view, Lucca appears (Figure 5) to be characterized by a general outward shifting of centrality, which in the last decades has gone, leaving the historic city to include the sprawled areas around the urban edge so as to progressively empty its inner centre. Here the purpose should be to promote easy access to the urban area encircled within the town walls, increasing the presence of parking and transport terminals in their close proximity. The density of touristic flows are hence expected to favour the persistency of economic activities and the survival of the ancient centre itself.

\section{Conclusions}

The results so far can be briefly summarized as follows.

In general a configurational approach appears to be a valuable key for taking into account the role of urban space in tourism management. The results of our case studies have proved that it is a reliable and suitable tool for supporting, 


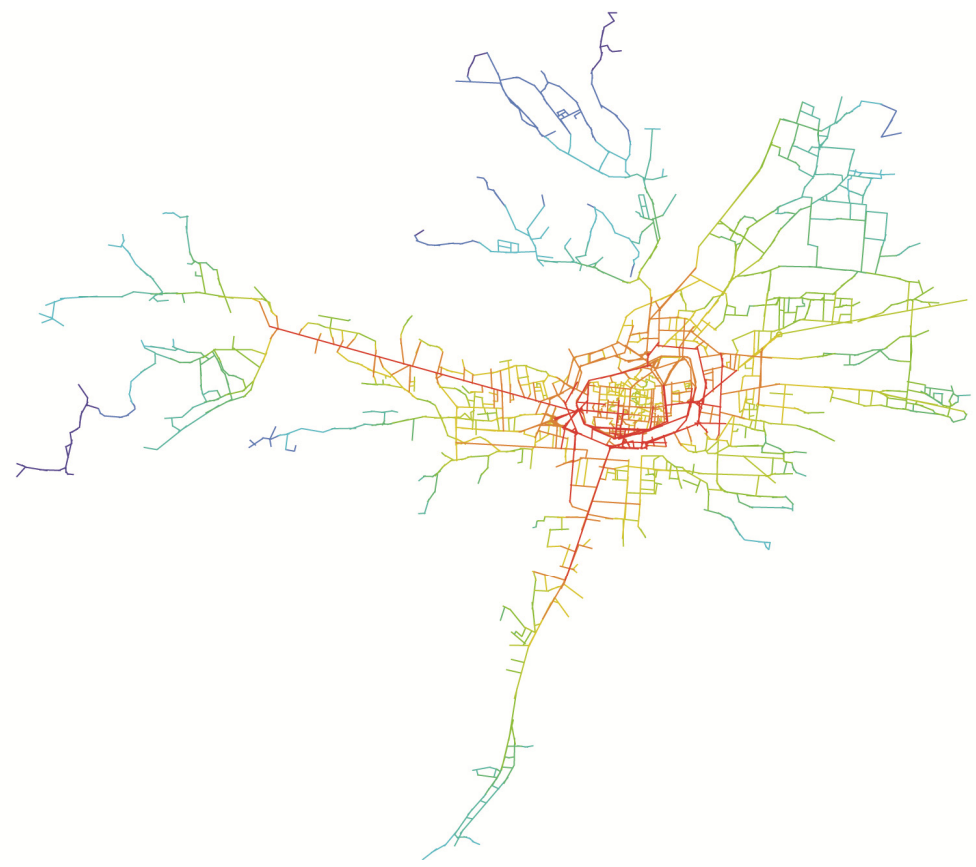

Figure 5: The distribution of global integration value in Lucca.

improving and managing the touristic accessibility of historic centres. Furthermore, the configurational state of an urban grid concisely reproduces its actual intelligibility, thus proving that there is a real need for tourist information and services. Moreover, the distribution of configurational indices can support the supply and location of information points and facilities, making them able to cope with the actual distribution of tourist flows. Furthermore, it can also be useful for managing touristic usage of historic cities in order to match the visit of places with the presence and operation of local activities, so as to favour actual tourism sustainability.

\section{References}

[1] Lew, A. \& McKercher, B., Modeling tourist movements. A local destination analysis. In: Annals of Tourist Research, vol. 33, pp. 403-423, 2006.

[2] Blank, U. \& Petkovich, M., The metropolitan area: a multifaceted travel destination complex. In: Tourism Planning and Development Issues, Washington University, pp. 393-405, 1987.

[3] Cutini, V. \& Rabino, G., Searching for Ariadne's thread. Some remarks on urban resilience and orientation. In: TEMA, vol. 5, pp. 7-22, 2012.

[4] Rabino, G. \& Caglioni, M., Ontology and M.A.S. of pedestrian behavior: the case study of tourists in Lucca. In: COST Action TU0801 Workshop, Skopje, Macedonia, 2012. 
[5] Hillier, B., Space is the Machine: a Configurational Theory of Architecture, Cambridge University Press: Cambridge (UK), 1996.

[6] Hillier, B. \& Hanson, J., The Social Logic of Space, Cambridge University Press: Cambridge (UK), 1984.

[7] Hillier, B., Penn, A., Hanson, J., Grajevski, T. \& Xu, J., Natural movement: or, configuration and attraction in urban pedestrian movement. In: Environment and Planning B, Planning and Design, vol. 20, pp. 67-81, 1993.

[8] Turner, A., Doxa, M., O’Sullivan, D. \& Penn, A., From isovists to visibility graphs: a methodology for the analysis of architectural space. In: Environment and Planning B: Planning and Design, vol. 28, pp. 103-121, 2001.

[9] Turner, A., Angular Analysis. In: Proceedings of the 3rd Space Syntax Symposium, Atlanta (GA), 7-11 May 2001, Alfred Tauban College of Architecture: University of Michigan, pp. 30.1-30.11, 2001.

[10] Hillier, B. \& Iida, S., Network and psychological effects in urban movement. In: Cohn, A. G. \& Mark, D. M. (eds) Spatial Information Theory: COSIT 2005, Lecture Notes in Computer Science, vol. 3693, 475-490, Berlin: Springer-Verlag, 2005.

[11] Cutini, V., Petri, M. \& Santucci, A., From axial maps to Mark Point Parameter Analysis (Ma.P.P.A.). A G.I.S. implemented method to automate configurational analysis. In: Computational Science and its Applications - ICCSA 2004 - Lecture Notes in Computer Science, vol. 3044, pp. 1107-1116, 2004.

[12] Cutini, V., Grilling the Grid: a Non-Ultimate (Nor Objective) Report on the Configurational Approach to Urban Phenomena. In: The Dynamics of Complex Urban Systems, Physica-Verlag: Heidelberg, pp. 163-183, 2007.

[13] Cutini, V., Managing Accessibility - The Configurational Approach to the Inclusive Design of Urban Spaces. In: Journal of Civil Engineering and Architecture, vol. 6, pp. 444-456, 2012.

[14] Tzortzi, K., Space. Interconnecting Museology and Architecture. In: The Journal of Space Syntax, vol. 2, pp. 26-53, 2011.

[15] Hillier, B. \& Tzortzi, K., Space Syntax: the language of museum space. In: McDonald, S., (Ed.) A Companion to Museum Studies, pp. 282-301, Blackwell Publishing Ltd:: Oxford (UK), 2006.

[16] Tzortzi, K., Museum building design and exhibition layout: patterns of interaction. In: Proceedings of the $6^{\text {th }}$ Space Syntax Symposium, pp. 72.1-72.16, 2007.

[17] Cutini, V., Centrality and Land Use: Three Case Studies on the Configurational Hypothesis. In: Cybergeo, Revue Européenne de Geographie, n. 188, 26 mars 2001.

[18] Coda, A., L'analisi configurazionale a supporto dei servizi turistici. Una sperimentazione su Venezia, Master of Science Thesis in Environmental and Land Planning Engineering, Politechnic of Milan, 2012. 